\title{
Negative Covariation between Task-related Responses in Alpha/ Beta-Band Activity and BOLD in Human Sensorimotor Cortex: an EEG and fMRI Study of Motor Imagery and Movements
}

\author{
Han Yuan ${ }^{1}$, Tao Liu ${ }^{3}$, Rebecca Szarkowski ${ }^{1}$, Cristina Rios ${ }^{1}$, James Ashe ${ }^{2,3}$, and Bin $\mathrm{He}^{1,2,{ }^{*}}$ \\ ${ }^{1}$ Department of Biomedical Engineering, University of Minnesota \\ 2 Department of Neuroscience, University of Minnesota \\ ${ }^{3}$ Brain Sciences Center, Veterans Affairs Medical Center, Minneapolis, MN
}

\begin{abstract}
Similar to the occipital alpha rhythm, electroencephalographic (EEG) signals in the alpha- and betafrequency bands can be suppressed by movement or motor imagery and have thus been thought to represent the "idling state" of the sensorimotor cortex. A negative correlation between spontaneous alpha EEG and blood-oxygen-level-dependent (BOLD) signals has been reported in combined EEG and fMRI (functional Magnetic Resonance Imaging) experiments when subjects stayed at the resting state or alternated between the resting state and a task. However, the precise nature of the task-induced alpha modulation remains elusive. It was not clear whether alpha/beta rhythm suppressions may covary with BOLD when conducting tasks involving varying activations of the cortex. Here, we quantified the task-evoked responses of BOLD and alpha/beta-band power of EEG directly in the cortical source domain, by using source imaging technology, and examined their covariation across task conditions in a mixed block and event-related design. In this study, 13 subjects performed tasks of right hand, right foot or left hand movement and motor imagery when EEG and fMRI data were separately collected. Task-induced increase of BOLD signal and decrease of EEG amplitudes in alpha and beta bands were shown to be co-localized at the somatotopic sensorimotor cortex. At the corresponding regions, the reciprocal changes of the two signals co-varied in the magnitudes across imagination and movement conditions. The spatial correspondence and negative covariation between the two measurements was further shown to exist at somatotopic brain regions associated with different body parts. These results suggest an inverse functional coupling between task-induced changes of BOLD and low-frequency EEG signals.
\end{abstract}

\section{Keywords}

EEG; BOLD; source imaging; desynchronization; spectral change

\footnotetext{
*Correspondence: Bin He, Ph.D., University of Minnesota, Department of Biomedical Engineering, 7-105 NHH, 312 Church Street, SE, Minneapolis, MN 55455, USA, binhe@umn.edu, Phone: 612-626-1115, Fax: 612-626-1114.

Publisher's Disclaimer: This is a PDF file of an unedited manuscript that has been accepted for publication. As a service to our customers we are providing this early version of the manuscript. The manuscript will undergo copyediting, typesetting, and review of the resulting proof before it is published in its final citable form. Please note that during the production process errors may be discovered which could affect the content, and all legal disclaimers that apply to the journal pertain.
} 


\section{Introduction}

Oscillatory activity has been a hallmark of human electroencephalographic (EEG) signals ever since Berger first reported a surge of scalp potential in the alpha $(8-12 \mathrm{~Hz})$ band when subjects closed their eyes (Berger, 1929). Such spontaneous oscillations can also be modulated by sensory, motor and cognitive tasks. A suppression of alpha and beta $(13-30 \mathrm{~Hz})$ activity detected at the central region of the scalp has been shown to be associated with movements and motor imagery (for review, see Pfurtscheller and Lopes da Silva, 1999). These task-related changes measured by EEG or magnetoencephalography (MEG), have been interpreted to be induced by the changes in synchrony of the underlying neuronal populations and are usually termed Event-related Desynchronization (ERD) or Event-related Synchronization (ERS) (Pfurtscheller and Lopes da Silva, 1999). However, the precise nature of the task-induced changes in neural oscillations is still poorly understood.

Combined EEG/MEG and fMRI (functional Magnetic Resonance Imaging) experiments have been conducted to investigate the physiological correlates of alpha activity (Fox et al., 2007; $\mathrm{He}$ and Liu, 2008). Through the neurovascular coupling, the blood-oxygen-level-dependent (BOLD)-fMRI signal is related to the composite neural activity with a complex organization in time and frequency. A tight positive correlation between the BOLD signal and synaptic activity reflected in gamma-band $(40-130 \mathrm{~Hz})$ local field potentials (LFP) has been suggested in simultaneously measured electrophysiological recordings and MR signals (Logothetis et al., 2001). On the contrary, an inverse correlation was demonstrated in a study that compared the LFP in low frequency bands $(5-15 \mathrm{~Hz})$ and the hemodynamic response to continuous auditory stimuli (Mukamel et al., 2005). Such negative correlation was corroborated in several studies comparing the EEG alpha rhythm and simultaneously measured hemodynamic signals when subjects closed their eyes and they demonstrated that the spontaneous modulations during the resting state were coupled (Goldman et al., 2002; Moosmann et al., 2003). A negative correlation of resting-state alpha activity with BOLD signals suggests that there may also be a negative correlation between task-induced responses of BOLD and EEG rhythm. In this regard, similar correlation between the two modalities in a task-on-task-off manner was observed in the visual area (Feige et al., 2005) and the sensorimotor area (Ritter et al., 2009) when subjects alternated between the resting state and one task. It was suggested that alpha rhythm and BOLD are a pair of antagonistic indicators for the neural activation, which can be combined as a very promising imaging approach for probing the brain function in high spatial and temporal resolution. However, the interpretation of the coupling was influenced by the finding that the spontaneous fluctuations during the resting state and task-evoked activity in BOLD signals can be linearly superimposed (Fox et al., 2006). Therefore, it is possible that a negative correlation with resting-state signal could override the correlation with task-related changes, if any.

These observations lead to an important question: are task-related changes in this pair of antagonistic indicators co-varied when conducting multiple tasks involving varying levels of activations of the same cortex? Specifically, we are interested in EEG alpha/beta suppressions and BOLD activations modulated by multiple tasks, which is different compared with previous study on resting conditions or alteration between resting conditions and one task. Given their inverse temporal correlation, there are two hypothesized relationships across task levels: (1) one signal remains proportional to task intensity while the other varies only in an on-off fashion; (2) the two signals show co-varied, proportional changes. Recent EEG/MEG/fMRI studies investigated the locations of alpha/beta decrease induced by cognitive (Singh et al., 2002) and motor tasks (Babiloni et al., 1999; Taniguchi et al., 2000; Winterer et al., 2007). They showed that movement-related EEG/MEG changes and BOLD increases overlapped spatially, whereas there was no correlation in the variable magnitudes across subjects within one condition (Winterer et al., 2007). However, a recent study reported that in the areas of the default mode 
network, including the visual/occipital cortex, the BOLD modulation was associated with different EEG-vigilance stages (Olbrich et al., 2009). In addition to the negative temporal correlation, decrease of BOLD signals was found to be related with increase of EEG alpha power when comparing the high vigilance stage with the low stage. This study suggested the latter hypothesized coupling relationship - a negative covariance across conditions between EEG alpha power and BOLD; however, the EEG alpha power suppressions in the sensorimotor areas co-varying with different levels of neural activations as reflected by BOLD signals need to be directly evidenced.

In addition, current available calculations of temporal correlation among EEG and BOLD usually compare the BOLD time course with the convolution of a kernel function (e.g. a double gamma function, Friston et al., 1998) and EEG power derived from one or a few electrodes placed at the scalp. Regarding this approach, there are at least two questionable limitations: 1) how to choose convolution kernel functions, which are used to unify the temporal scales of EEG (millisecond level) and BOLD signals (second level) and vary significantly due to many reasons, particularly in the case of alpha rhythm; 2) how to choose temporal waveforms of EEG to be convoluted with the kernel function since surface EEGs are possibly contributed by many sources originating from different neural substrates. Although some studies have implemented different spatial or temporal de-convolution technologies (Ritter et al., 2009; de Munck et al., 2009), they are not able to fundamentally overcome these two limitations. Towards this end, there is a need to develop an approach co-registering the disparate spatial and temporal scales of EEG and BOLD when investigating their coupling relationship.

Here we propose to investigate the relationship between task-related changes in alpha/beta EEG and BOLD responses during motor imagery and movement. We developed a mixed block and event-related design to facilitate the analysis of fMRI and EEG signals at different temporal scales. EEG and fMRI data were collected in separate sessions but under identical stimuli. We then employed source analysis in the frequency domain to compare the task-evoked changes in EEG with the BOLD responses in target cortical areas. In the present study, we addressed the following questions:

1. Are task-related alpha/beta changes co-localized with BOLD responses?

2. If so, do alpha/beta changes co-vary with BOLD responses across task conditions?

3. If so, does the relationship exist at different cortical areas specific to different body parts?

\section{Materials and Methods}

\section{Subjects}

Thirteen healthy subjects (ages $19-45$ years, $24.2 \pm 8.0$ years, six males) participated in the study with written consent according to a protocol approved by the Institutional Review Board of the University of Minnesota. Twelve subjects were right-handed and one subject was ambidextrous as measured by the Edinburgh Handedness Inventory (Oldfield, 1971).

\section{Experimental Design}

A mixed block and event-related design was developed to accommodate the EEG and fMRI recordings, as shown in Fig. 1. The blocks of task conditions were interleaved with rest blocks. Each block lasted for $20 \mathrm{~s}$. Within a task block, there were six events during which subjects performed the appropriate task for $2 \mathrm{~s}$ (imagination or movement execution) interleaved with inter-trial intervals of varying durations from $1 \mathrm{~s}$ to $2 \mathrm{~s}$. The same tasks were performed within a block and the sequence of block types was randomized and balanced across runs and subjects. 
There were four motor imagery conditions (left hand, right hand, right foot and tongue) and four conditions during which subjects performed the actual movements. Results regarding tongue imagination and movement were not significantly identified and thus not included here. Visual cues were presented to indicate the conditions of events. During the movement conditions (designated as Move Right, Move Left and Move Foot), subjects repeatedly fully clenched their left or right hand or toes at a pace of $3 \mathrm{~Hz}$. For the imagery conditions (designated as Imagine Right, Imagine Left and Imagine Foot), subjects were instructed to imagine that they were clenching their left or right hand or toes at the same pace. A metronome beating at $3 \mathrm{~Hz}$ was constantly active during the entire run to pace the movements and imaginations. Subjects kept their eyes open for all sessions.

In order to control the absence of movement during the imagination tasks, substantial training and monitoring were conducted for the EEG and fMRI recordings. For each subject, the EEG experiments were performed before the fMRI sessions. In EEG sessions, subjects went through sufficient training before data recording till they can appropriately perform the instructed tasks, while their movements were monitored by the online inspection of EMG signals and also visual inspection by researchers. In fMRI sessions, training was conducted again prior to subjects entering the scanner.

\section{Data Acquisition}

EEG signals and fMRI images were collected during separate sessions. EEG activity was recorded from 62 electrodes positioned according to the international 10-20 system (BrainCap, Brain Products $\mathrm{GmbH}$, Germany) when subjects sat in a comfortable armchair in an electrically shielded room. The signals were referenced to a point at the vertex at position $\mathrm{FCz}$ and acquired using a BrainAmp MR amplifier (BrainProducts GmbH, Germany) with an analog $200 \mathrm{~Hz}$ low-pass $/ 0.1 \mathrm{~Hz}$ high-pass filter at a sampling frequency of $1000 \mathrm{~Hz}$. The physical landmarks (nasion and left, right preauricular points) and electrode positions were digitized using a Polhemus Fastrak digitizer (Polhemus, Colchester, VT) and 3DSpace software from the SCAN software package (NeuroScan Labs, TX). Electromyogram (EMG) activity was also recorded in the EEG sessions to monitor muscle movement by a Synaps2 amplifier (NeuroScan Labs, TX) at a sampling frequency of $2000 \mathrm{~Hz}$. Three pairs of bi-polar EMG channels were used to capture the movements of left hand, right hand and right foot. The hand EMG channels were placed at the ventral side of each forearm approximately two inches from the wrist. And the foot EMG channels were placed at the dorsal middle surface of the right foot approximately three inches from the roots of toes. For all three channels, bursts of EMG signals were clearly identified to be associated with each movement while no EMG bursts were present during imagination periods.

Each individual anatomical MRI data set consisted of 176 contiguous sagittal slices with $1 \mathrm{~mm}$ slice thickness (matrix size: $256 * 256$; FOV: $256 \mathrm{~mm} * 256 \mathrm{~mm}$; TR/TE=20 ms $/ 3.3 \mathrm{~ms}$ ) on a 3T MRI system (Siemens Trio, Siemens, Erlangen, Germany). Whole-brain functional images with BOLD contrast were acquired using gradient echo planar imaging sequence (32 axial 3$\mathrm{mm}$ thick interleaved slices with $0.3-\mathrm{mm}$ gap; TR $/ \mathrm{TE}=2000 \mathrm{~ms} / 30 \mathrm{~ms}$; flip angle $=90^{\circ}$; matrix size: $64 * 64$; FOV: $192 \mathrm{~mm} * 192 \mathrm{~mm}$ ). Each functional run started with a rest block and contained 330 volumes. Three runs were collected for each subject.

\section{EEG Analysis}

EEG recordings were band-pass filtered from $1-30 \mathrm{~Hz}$ using a zero-phase FIR filter and segmented into epochs from $-1 \mathrm{~s}$ to $2.5 \mathrm{~s}$ with respect to the onset of visual cues for tasks. Then, each epoch was baseline corrected and detrended. Epochs with eye movements were visually identified and excluded from further analysis. The artifact-free signals were down- 
sampled to $200 \mathrm{~Hz}$. The EMG recordings were high-pass filtered at $10 \mathrm{~Hz}$, rectified, and segmented along with the EEG signals.

The time-frequency representations (TFR) of single-trial EEG signals were calculated using Complex Morlet's wavelet. The EEG signal, $x(t)$, was first convoluted by wavelets,

$\omega(t, f)=A \cdot \exp \left(-t^{2} / 2 \sigma_{t}^{2}\right) \cdot \exp (2 i \pi f t)$, with $A=\left(\sigma_{t} \pi^{1 / 2}\right)^{-1 / 2}, \sigma_{\mathrm{t}}=1 /\left(2 \pi \sigma_{f}\right)$, and $\sigma_{\mathrm{f}}=f / 7$. The tradeoff ratio $\left(f / \sigma_{\mathrm{f}}\right)$ was chosen as 7 to create a wavelet family (Tallon-Baudry et al., 1997; Qin et al., 2004). For each time $t$ and frequency $f$, the power of the EEG signal, $P(t, f)$, is the squared norm of the convolution: $P(t, f)=\left|\omega(t, f)^{*} x(t)\right|^{2}$. The normalized time-varying power of EEG was computed for visualization. It was characterized in $t$ statistic by contrasting the power at each time-frequency pair to the power at the same frequency before the task (from $-150 \mathrm{~ms}$ to $-50 \mathrm{~ms}$ ) using an unpaired $t$ test. The group time-frequency changes of all subjects was calculated using a random-effect model (one-sample Student's $t$-test) and thresholded with $p<0.05(|t|>2.18)$, as illustrated in Fig. 2. The group TFR plots were used to identify the time and frequency windows for the source analysis explained below. The task period was selected to be from $0.3 \mathrm{~s}$ to $1.9 \mathrm{~s}$ with respect to the onset of the event, during which steady decrease was observed in the group time-frequency changes. Meanwhile, the baseline period was from $-1 \mathrm{~s}$ to $-0.1 \mathrm{~s}$, during which little change was present. In addition to the time-frequency plots, the scalp topology of relative changes between task and baseline were also visualized. The time-varying power from the wavelet analysis was averaged in the alpha or beta frequency bands, and averaged in the time windows of task and baseline periods, respectively. The relative changes induced by tasks, i.e. the power difference between task and baseline conditions and normalized by the baseline power, were plotted according to their channel locations over the scalp.

The EEG source power was calculated using the Minimum Norm Estimate in Frequency Domain method (Yuan et al., 2008). The EEG sources were modeled as many dipoles distributed on the cortical surface and oriented perpendicular to the surface, as shown in the following linear equation:

$$
\Phi=A \cdot S+N
$$

where $\Phi$ is a matrix of the measured EEG, $S$ is the unknown matrix of amplitudes of the dipoles, and $N$ is the additive noise. $A$ is the transfer matrix, which relates between dipole sources and scalp potentials. The cortical surface reconstructed from individual subject's MRIs was used to restrict the source locations and orientations (Dale and Sereno, 1993). The minimum-norm estimate of sources, $S^{\prime}$, was obtained by applying a linear operator to the EEG measurements, using the following equation:

$$
S^{\prime}=R A^{T}\left(A R A^{T}+\lambda^{2} C\right)^{-1} \Phi,
$$

and

$$
\lambda^{2}=\frac{\operatorname{tr}\left(A R A^{T}\right)}{\operatorname{tr}(C) S N R^{2}}
$$

where $S N R$ is the signal to noise ratio, $C$ and $R$ are covariance matrices of the noise and sources, respectively (Yuan et al., 2008). In order to estimate the sources in the alpha and beta frequency bands, single-trial EEG epochs were first converted into the frequency domain using Fourier 
transformation. The real and imaginary parts were subjected to source estimation. The source power was obtained by selectively summing up the estimates in the frequency bins of the targeted bands. After the source estimates for task and baseline periods were separately calculated, we compared the distributions of source power for each task condition with the pooled baseline conditions. The task-related spectral changes of EEG source activity were quantified as the event-related increase or decrease relative to the baseline (i.e. the power difference between task and baseline conditions) and normalized by the baseline power.

All dipoles from each individual were then projected onto a reference brain by aligning each subject's sulcal-gyral patterns using BrainVoyager QX (Fischl et al., 1999; Goebel et al., 2006; Brain Innovation, Maastricht). The spectral changes were used to make a random-effect statistical map (one-sample Student's $t$-test) of the differential neural activity on the cortical surface. The group average maps were thresholded by $p<0.001(|t|>4.32, p$ uncorrected for multiple-comparison) for both imagery and movement.

\section{fMRI Analysis}

MRI data were analyzed using BrainVoyager (Brain Innovation, Maastricht). EPI images were coregistered with $\mathrm{T} 1$ anatomy and then transformed into the Talairach space. The preprocessing of functional data included motion correction, slice time correction, spatial smoothing using a Gaussian kernel of FWHM $=6 \mathrm{~mm}$ and temporal high pass filtering. The signal time course of each subject was modeled with a boxcar function convolved with a double-gamma hemodynamic response function (Friston et al., 1998). Independent regressors were set for different task conditions. The individual task-related activity was evaluated using a general linear model (GLM) (Friston et al., 1995). The resulting set of voxel values for each comparison constituted the map of beta weight of each regressor (task vs. baseline) and the statistical significance associated with each regressor. The magnitudes of beta values were proportional to the signal change between activation and baseline, which represented the magnitude of the BOLD response. GLM group-level analysis (random effect analysis, one-sample Student's $t$ test)) was performed based on the beta values from individual analysis. $t$ statistic maps were thresholded by $p<0.001$ ( $p$ uncorrected for multiple-comparison).

\section{Region of Interest Analysis}

Region of Interest (ROI) analysis was performed to investigate the cross-modal relationship between the EEG and fMRI responses across two experimental conditions (imagination and movement) and across subjects. Anatomical masks were created according to the Talairach atlas database (Lancaster et al., 2000), including the precentral, postcentral, and paracentral gyrus, all of which were shown to be activated by imagination and movement (Ehrsson et al., 2003). ROIs for imagination or movement of each body part were created based on the thresholded group maps of EEG and fMRI (task vs. baseline, $p<0.001$ ) that intersected with the anatomical masks. From the defined ROIs, the mean and individual percentage changes of EEG in alpha/beta band and the beta values of the GLM analysis were extracted (all $p<0.05$, after small-volume correction for multiple-comparison). For each subject, the EEG percentages were normalized by the maximum absolute change across all conditions. The BOLD beta values were normalized similarly as well. The normalized quantities associated with imagination and movement of the same body part was pooled and linear regression was conducted to assess the relation between EEG and BOLD responses. Separate regression analysis was performed for different EEG bands and at different areas specific to the body parts. 


\section{Results}

The experimental paradigm and analysis stream were illustrated in Fig. 1. EEG and fMRI data were separately collected in the same group of subjects using the same paradigm. The taskinduced changes of EEG and BOLD signals were quantified as relative change and beta values respectively and later compared to examine their covariation relations. An example of singletrial EEG traces was included in Fig. 1. The traces after filtering from $8 \mathrm{~Hz}$ to $12 \mathrm{~Hz}$ were also shown, demonstrating a profile of decreasing power accompanying the task period. Such timevarying change was visualized by time-frequency representations in Fig. 2(A-C). For trials of imagination tasks, the EMG during task period $(0.3 \mathrm{~s} \sim 1.9 \mathrm{~s})$ were compared to the EMG baseline activity ( $-1 \mathrm{~s} \sim-0.1 \mathrm{~s})$. For all subjects, no significant EMG activities were detected during the imagination (all $p>0.05$, paired Student's $t$ test). Significant EMG activities were present corresponding to the tasks of movement.

\section{Spatial Correspondence between EEG Power and fMRI BOLD Changes}

Fig. 2(A-C) shows the event-related spectral changes of EEG signals recorded from the $\mathrm{C} 3$, $\mathrm{C} 4$, and $\mathrm{Cz}$ electrode over the central region of the brain using wavelet analysis. A steady change was found from $0.3 \mathrm{~s}$ to $1.9 \mathrm{~s}$ during the task period and the source power was estimated from this window. Meanwhile, the baseline was selected from $-1 \mathrm{~s}$ to $-0.1 \mathrm{~s}$. The scalp distributions of relative change in the beta frequency band were shown in Fig. 2(D). For left or right hand task, the electrodes contralateral to the task side show a dominant suppression of power in the alpha $(8 \mathrm{~Hz}-12 \mathrm{~Hz})$ and beta $(13 \mathrm{~Hz}-28 \mathrm{~Hz})$ bands during the imagery and movement conditions. There was a significant decrease in alpha and beta power at the ipsilateral electrode during movement as well, but of a smaller magnitude compared to the contralateral electrode. For the foot task, a decrease was mainly observed at the Cz electrode accompanying both imagination and movement. The topological difference between tasks associated with left, right hand, and foot can be mainly observed at C3, C4 and Cz electrodes. Similar topology of EEG changes in the alpha band was included as Fig. 3 in the supplemental materials. No significant alpha change was found for the task of foot imagination.

Fig. 3(A-F) shows the task-related BOLD changes as well as EEG source changes in alpha and beta bands displayed on an inflated cortical surface for the imagination and movement of right hand. The decreases in EEG alpha and beta bands were depicted over the contralateral sensorimotor areas across the central sulcus. A more focused increase in BOLD was colocalized at the contralateral sensorimotor area around the motor-hand area (Yousry et al., 1997). For the maps of EEG changes during right hand movement, ipsilateral decrease was found at the approximately mirrored sensorimotor areas.

Similarly, for the imagination and movement of right foot, the areas with decrease of EEG beta activity largely overlapped with those with BOLD increase at the medial part of primary sensorimotor cortex (Fig 4, A-D). The changes of EEG alpha activity during movement of right foot were shown in Supplemental Fig. 3 (A-B), with decrease of alpha and increase of BOLD at the medial sensorimotor cortex. No significant changes in EEG alpha activity was identified at the threshold of $p<0.001(|t|>4.32$, uncorrected) or $p<0.05(|t|>2.18$, uncorrected).

The EEG and BOLD maps for imagination and movement of left hand were shown in Supplemental Fig. 1. Bilateral ERDs in the alpha and beta frequency bands were observed during the left hand movement. During the imagination of left hand, a dominant contralateral decrease of EEG alpha or beta activity was also found at the sensorimotor area. However, no significant activation of BOLD was identified for the imagination of left hand at the corresponding area at the threshold of $p<0.001$ (uncorrected). But at the threshold of $p<0.05$ (uncorrected), an overlapping but smaller region was identified in the BOLD map than the area 
of EEG change. The ipsilateral ERD induced by left-hand imagination was found only present in the beta frequency band but not in the alpha band.

The peak locations of EEG alpha, beta and BOLD changes are shown in Fig. 6. The maximum changes and corresponding coordinates are listed in Table-1. The peaks of BOLD, EEG alpha and beta changes were all located close to the central sulcus. The BOLD peaks for imagination were more anterior than those associated with movements. The average distances between alpha/beta changes and BOLD changes were $13.26 \pm 4.76 \mathrm{~mm} / 13.33 \pm 8.15 \mathrm{~mm}$ for imagination and movement, respectively. Tables of all activated regions identified from EEG and fMRI maps were listed in the supplemental materials, including the supplemental motor area (SMA).

\section{Negative Covariation between EEG Power and fMRI BOLD Changes}

Fig. 3(G-H) shows the relationship between EEG alpha/beta changes and BOLD changes from the contralateral ROI defined from thresholded group-maps during imagination and movement of right hand. A negative covariation relationship between EEG and BOLD changes was found using Pearson's correlation ( $p<0.0004$ for BOLD and EEG beta, and $p<0.005$ for BOLD and EEG alpha). At the foot-specific area of sensorimotor cortex, the EEG beta changes were also negatively covaried with BOLD changes ( $p<1 \mathrm{e}-5$, Fig. 4(E)).

The average changes of alpha, beta and BOLD across all conditions are shown in Fig. 5. Results showed decreased alpha or beta changes were associated with increased BOLD responses when comparing the movement condition with the imagination at each somatotopic region. No significant regions associated with imagination of left hand were identified in BOLD maps. And no areas were found for imagination of foot in EEG alpha maps. Therefore, it was not feasible to pool the quantities of imagination and movement between EEG alpha and BOLD at the region of foot, or between EEG alpha/beta and BOLD at the region of left hand. The regression from only one task condition was largely dependent on the inter-subject variation rather than across-task variation and was not significant (Supplemental Fig $1(\mathrm{~F}-\mathrm{G})$ and Supplemental Fig $2(\mathrm{C})$ ).

The quantified changes of different body parts were also pooled together to test the hypothesis that the changes associated with movement were different from those with imaginations. The results showed that the changes in both EEG and BOLD associated with movement were larger in magnitude than those associated with imagery ( $p=3 \mathrm{e}-5$ for alpha changes, $p=2 \mathrm{e}-11$ for beta changes, and $p=1 \mathrm{e}-11$ for BOLD changes, paired one-side $t$ test).

\section{Discussion}

The primary aim of the current study was to enhance our understanding of the biophysical relationship between task-related EEG changes and hemodynamic responses, particularly to examine the covariation of EEG and fMRI responses across multiple task conditions. We collected EEG and fMRI data in separate sessions using a mixed block and event-related design to investigate the alpha/beta EEG and BOLD responses during motor imagery and movement. Our results showed that: (1) the contralateral decrease of alpha/beta EEG is co-localized with an increase of BOLD in the primary sensorimotor cortex; (2) across different task levels, the magnitudes of EEG changes co-varied with BOLD responses; and (3) the spatial correspondence and negative covariation existed at different somatotopic cortical areas associated with different body parts.

\section{Co-variation of Task-related Changes of EEG and BOLD Responses}

Recent studies have demonstrated the coupling between hemodynamic signals and lowfrequency neurophysiologic oscillations, in the spontaneous resting state (Goldman et al., 
2002; Moosmann et al., 2003) and in an alternation between the resting state and one task

(Feige et al., 2005; Ritter et al., 2009). However, the temporal correlation in a task-on-task-off manner is not necessarily the same as the covariation of responses across multiple task levels. Our results for the first time demonstrated the coupling covariation between the two imaging modalities, which is of critical importance to prove that the alpha/beta suppression also contains the rich information about the strength of cortical activity induced by tasks. This study extends the previous event-related de-synchronization (ERD) research as most of them focused on localization. While the increase of BOLD and decrease of alpha/beta-band amplitudes were co-localized, our results showed that their magnitudes of changes were proportional across task conditions and across subjects. Decreased alpha or beta changes were associated with increased BOLD responses when comparing the movement condition with the imagination at the sensorimotor cortex. Interestingly, this finding is corroborated with a parallel study by Olbrich et al. in the visual/occipital area about the BOLD and EEG response to different vigilance stages (2009). They reported a more decreased BOLD signals associated with increased alpha activity between low and high vigilance conditions, on top of their inverse correlation. These results imply that both occipital and sensorimotor alpha/beta rhythms are functionally coupled with the BOLD signals, possibly through a common mechanism. Furthermore, our results demonstrated that the spatial correspondence of reciprocal changes and negative covariation was found to be located at somatotopic regions specific to the performing limb in the primary sensorimotor area, which further suggests the functional coupling relationship.

In our method, we integrated EEG source imaging technology together with the covariation investigation study between EEG and BOLD signals. Compared with other studies which correlated the BOLD time course with a convolution of a kernel function and the EEG from one or a few electrodes, we directly used EEG source power from EEG source imaging results, which solved the mismatch of spatial scales. Since we quantified both the BOLD and alpha responses as the relative changes (task vs. baseline) and it was based on our unique mixed design of block and event-related tasks, we avoided to address the large temporal scale difference between EEG and BOLD. Through co-registering the spatial and temporal scales of alpha and BOLD signals, our study is believed to accurately capture the relationship between task-induced responses.

Our current results are in line with the findings at the primary visual/auditory cortex where the increase of gamma-band amplitudes was shown to be positively correlated with the increase of BOLD signals under multiple stimulus conditions (Logothetis et al., 2001; Hall et al., 2005), which coincided with the positive temporal correlation during stimulus (Logothetis et al., 2001; Mukamel et al., 2005). Interestingly, the decrease of alpha power was co-localized with BOLD response and gamma power increase in a visual stimulation paradigm (Brooks et al., 2005). In another comparison of BOLD response and MEG oscillations in a motor task, Winterer et al. found that a complicated pattern of increase or decrease at several frequency bands accompanying BOLD responses can be found at the same cortical areas (Winterer et al., 2007). Taken together, these data suggest that the hemodynamic response can be linked to various aspects of the changes in neural activity reflected at different frequency bands. Particularly, since it is easier and more accurate to detect EEG alpha rhythm, as compared with gamma rhythm, it is promising to have more applications in imaging the brain functions using EEG or combined EEG-fMRI modalities.

\section{Localization of Task-related Decrease of EEG in Alpha- and Beta-frequency Bands}

Our results demonstrated a somatotopic co-localization between decrease in EEG alpha/beta activity and increase of BOLD in imagination and movement of right hand, right foot and left hand. In particular, our results for the first time reported the EEG source results regarding foot imagery and movement and its co-localization with fMRI maps. Motor imagery has been 
widely used in human Brain-Computer Interface (BCI) studies as a mental strategy for prosthetic control (Wolpaw and McFarland, 2004). The Rolandic alpha rhythm at the central regions (also known as mu rhythm) and beta rhythms can be intentionally modulated by motor imagery (Pfurtscheller and Lopes da Silva, 1999). Previous studies have investigated the EEG sources underlying the changes in the alpha- and beta-frequency bands during motor imagery alone (Kamousi et al., 2005; Kamousi et al., 2007; Qin et al., 2004) and with feedback derived from online classification of imagery tasks (Yuan et al., 2008). The primary sensorimotor area was found to be the neural origin of the event-related decrease associated with motor imagery, which is consistent with our present results. Furthermore, we demonstrate here that a hemodynamic response likely driven by neural synaptic activity is correlated with the modulation of EEG rhythms, which indicates a neurophysiologic basis for the BCI control.

At the sensorimotor areas, the imagination of left hand was found to be associated with bilateral decrease of EEG power in the beta frequency band. Bilateral ERD in the beta band was also shown accompanying the movement of left/right hand. Furthermore, the bilateral difference in the beta band during the imagination of left hand was not significantly different with that during movement of left hand $(p>0.05)$. However, in the alpha band, only contralateral ERD was identified for the imagination of left hand, whereas bilateral alpha ERD was present for the left-hand movement, which indicates that alpha ERD shows stronger lateral dominance during left-hand imagination. The increase of alpha activity (ERS) was found only in a few subjects for the imagination tasks, which however, was not significant at the group level (random-effect analysis, $p>0.05$ in Supplemental Fig. 3, $p>0.001$ in Fig. 3 and Supplemental Fig. 1). This is consistent with the study of ERD/ERS in nine human subjects by Pfurtscheller et al., who reported large subject variability on the alpha ERS associated with imaginations whereas the ERD patterns appeared to be more consistent among subjects (2006).

The co-localization of alpha/beta EEG decrease with BOLD increase during movement is consistent with previous EEG/MEG studies in which a decrease of alpha and beta rhythm was located in the vicinity of the central sulcus during voluntary movement (Salmelin et al., 1995; Babiloni et al., 1999; Taniguchi et al., 2000; Jurkiewicz et al., 2006) and tactile stimulation (Gaetz and Cheyne, 2006). Electrocorticographic recordings from epileptic patients undergoing pre-surgical monitoring have also shown a decrease of power in alpha and beta bands at electrodes placed over the contralateral peri-central gyri (Crone et al., 1998b; Miller et al., 2007). In addition to the sensorimotor areas, our results found significant activation of SMA in fMRI maps associated with both imagination and movement task conditions, except the imagination of left hand. EEG maps also showed significant decrease at SMA in a few conditions, which overlapped with the fMRI activated regions. These findings point in the same direction as several previous MEG/fMRI studies which reported that a decrease of EEG rhythms was accompanied by positive BOLD responses during visual tasks (Brookes et al., 2005), visuomotor discrimination tasks (Winterer et al., 2007), and cognitive tasks (Singh et al., 2002). It is worth noting that an increase in the high frequency gamma range accompanying a decrease in low frequency (alpha/beta) activity was found in the contralateral sensorimotor cortex during ECoG recordings (Crone et al., 1998a; Miller et al., 2007). An analogous result was also reported to occur in the visual cortex where BOLD increase was colocalized with a decrease in alpha and an increase in gamma in response to visual stimuli (Brookes et al., 2005; Muthukumaraswamy and Singh, 2009). These results imply that highand low-frequency band changes might arise from different physiological phenomena. They also suggest that the increase of BOLD signal, the decrease of EEG/ECoG (and other electrophysiological signals sampling the common sources) in low-frequency band, and the increase of EEG/ECoG in high-frequency band can be related in the same context of taskevoked responses. 


\section{Functional Interpretation for Task-related EEG Changes}

BOLD signals depend on CBF, CBV and metabolic consumption of oxygen that are closely related to neuronal activities. Coupling between electrophysiological and hemodynamic changes allows for high-resolution functional mapping as well as inferences about brain function. A linear correlation between BOLD signals and neuronal activity was demonstrated by Logothetis et al. who simultaneously acquired intra-cortical electrophysiological and fMRI data in a monkey's visual cortex (Logothetis et al., 2001). Their results showed that synaptically generated local field potentials and multi-unit activity were reliable predictors of BOLD response, indicating that the stimulus-evoked increase of the BOLD signal represents increased neuronal activity. Local field potentials are thought to reflect the weighted average of the synchronized dendrosomatic components of pyramidal synaptic signals and share a common source with synchronized electromagnetic signals recorded from the scalp (EEG/MEG) (Hamalainen et al., 1993; Logothetis and Wandell, 2004). Similarly, a correlation with the positive BOLD response was also reported from scalp electromagnetic recordings, including surface-negative slow cortical potentials (Hinterberger et al., 2003; Nagai et al., 2004) and event-related potentials (ERP) through phase-locked averaging (Huettel et al., 2004; Mulert et al., 2004; Schulz et al., 2004; Babiloni et al., 2005; Liu and He, 2008) - all of which are generated by synchronized neuronal populations.

However, our results demonstrated an inverse relationship between the BOLD response and task-evoked changes in the alpha frequency band, which is thought to arise from synchronization of postsynaptic potentials across cortical neuronal populations (Nunez et al., 2001; Pfurtscheller and Lopes da Silva, 1999). Such negative correlations imply that the taskinduced changes of low-frequency band EEG and ERP reflecting phase-locked activity may arise from different physiological phenomena, whereas it is not clear how the synaptic activity of neuronal populations would change in relation to task-related changes in oscillation, and hence a hemodynamic change. Previous studies found that synaptic networks of neurons in isolated slices of neocortex may generate and maintain synchronized oscillations in the alpha frequency band (Silva et al., 1991), which were also demonstrated in the visual cortex of dog using intracortical recordings (Lopes da Silva and Storm van Leeuwen, 1977). In response to certain tasks, neuronal activation may change the cortico- and/or thalamo-cortical interactions between neuronal populations that control the frequency components of the ongoing oscillations, and thus lead to the change of EEG alpha/beta activity or the hemodynamic response through the interplay of neurovascular coupling, as suggested by our results. The finding of the inverse correlation is also compatible with the notion that low-frequency electrophysiological signals indicate an "idling state" of brain activity, as decrease of alpha activity is linked to neural activation suggested by BOLD response. The decrease in alpha/beta activity may be due to a decrease in the synchrony of the underlying neural populations. In particular, our results suggest that the degree of desynchronization may be quantitatively related with increased neuronal activity, as reflected in the increased BOLD signal.

\section{Supplementary Material}

Refer to Web version on PubMed Central for supplementary material.

\section{Acknowledgments}

The authors would like to thank Dr. Zhongming Liu, Christopher Wilke and Audrey Royer for helpful discussions, and Megan Savage for assistance in collecting the experimental data in the subjects. The authors are also grateful to anonymous reviewers for their constructive comments. This work was supported in part by NIH RO1EB007920, NIH RO1EB006433, and NSF CBET-0933067. H.Y. was supported in part by an NIH Neuro-Physical-Computational Sciences Fellowship R90 DK070106. The 3T MRI scanner was partially supported by NIH P41RR008079 and P30NS057091. 


\section{References}

Berger H. Uber das Elektroenkephalogramm des Menschen. Arch Psychiatr Nervenkr 1929;87:527-570.

Brookes MJ, Gibson AM, Hall SD, Furlong PL, Barnes GR, Hillebrand A, Singh KD, Holliday IE, Francis ST, Morris PG. GLM-beamformer method demonstrates stationary field, alpha ERD and gamma ERS co-localisation with fMRI BOLD response in visual cortex. NeuroImage 2005;26:302-308. [PubMed: 15862231]

Babiloni C, Carducci F, Cincotti F, Rossini PM, Neuper C, Pfurtscheller G, Babiloni F. Human movement-related potentials vs desynchronization of EEG alpha rhythm: a high-resolution EEG study. Neuroimage 1999;10:658-665. [PubMed: 10600411]

Babiloni F, Cincotti F, Babiloni C, Carducci F, Mattia D, Astolfi L, Basilisco A, Rossini PM, Ding L, Ni Y, Cheng J, Christine K, Sweeney J, He B. Estimation of the cortical functional connectivity with the multimodal integration of high-resolution EEG and fMRI data by directed transfer function. Neuroimage 2005;24:118-131. [PubMed: 15588603]

Crone NE, Miglioretti DL, Gordon B, Lesser RP. Functional mapping of human sensorimotor cortex with electrocorticographic spectral analysis. II. event-related synchronization in the gamma band. Brain 1998a;121:2301-2315. [PubMed: 9874481]

Crone NE, Miglioretti DL, Gordon B, Sieracki JM, Wilson MT, Uematsu S, Lesser RP. Functional mapping of human sensorimotor cortex with electrocorticographic spectral analysis. I. alpha and beta event-related desynchronization. Brain 1998b;121:2271-2299. [PubMed: 9874480]

Dale AM, Sereno MI. Improved localization of cortical activity by combining EEG and MEG with MRI cortical surface reconstruction: a linear approach. Journal of Cognitive Neuroscience 1993;5:162-176.

de Munck JC, Gonçalves SI, Mammoliti R, Heethaar RM, Lopes da Silva FH. Interactions between different EEG frequency bands and their effect on alpha-fMRI correlations. NeuroImage 2009;47:6976. [PubMed: 19376236]

Ehrsson HH, Geyer S, Naito E. Imagery of voluntary movement of fingers, toes, and tongue activates corresponding body-part-specific motor representations. Journal of Neurophysiology 2003;90:33043316. [PubMed: 14615433]

Feige B, Scheffler K, Esposito F, Di Salle F, Hennig J, Seifritz E. Cortical and subcortical correlates of electroencephalographic alpha rhythm modulation. Journal of Neurophysiology 2005;93:28642872. [PubMed: 15601739]

Fischl B, Sereno MI, Tootell RB, Dale AM. High-resolution intersubject averaging and a coordinate system for the cortical surface. Human Brain Mapping 1999;8:272-284. [PubMed: 10619420]

Fox MD, Raichle ME. Spontaneous fluctuations in brain activity observed with functional magnetic resonance imaging. Nature Reviews. Neuroscience 2007;8:700-711. [PubMed: 17704812]

Fox MD, Snyder AZ, Zacks JM, Raichle ME. Coherent spontaneous activity accounts for trial-to-trial variability in human evoked brain responses. Nature Neuroscience 2006;9:23-25.

Friston KJ, Holmes AP, Worsley KJ, Poline J, Frith CD, Frackowiak RSJ. Statistical parametric maps in functional imaging: A general linear approach. Human Brain Mapping 1995;2:189-210.

Friston KJ, Fletcher P, Josephs O, Holmes A, Rugg MD, Turner R. Event-related fMRI: Characterizing differential responses. NeuroImage 1998;7:30-40. [PubMed: 9500830]

Gaetz W, Cheyne D. Localization of sensorimotor cortical rhythms induced by tactile stimulation using spatially filtered MEG. NeuroImage 2006;30:899-908. [PubMed: 16326116]

Goebel R, Esposito F, Formisano E. Analysis of functional image analysis contest (FIAC) data with brainvoyager QX: From single-subject to cortically aligned group general linear model analysis and self-organizing group independent component analysis. Human Brain Mapping 2006;27:392-401. [PubMed: 16596654]

Goldman RI, Stern JM, Engel J Jr, Cohen MS. Simultaneous EEG and fMRI of the alpha rhythm. Neuroreport 2002;13:2487-2492. [PubMed: 12499854]

Hall SD, Holliday IE, Hillebrand A, Singh KD, Furlong PL, Hadjipapas A, Barnes GR. The missing link: analogous human and primate cortical gamma oscillations. NeuroImage 2005;26:13-17. [PubMed: 15862200] 
Hamalainen MS, Hari R, Ilmoniemi RJ, Knuutila J, Lounasmaa OV. Magnetoencephalography - theory, instrumetation, and applications to noninvasive studies of the working human brain. Reviews of Modern Physics 1993;65:413-497.

He B, Liu Z. Multimodal Functional Neuroimaging: Integrating Functional MRI and EEG/MEG. IEEE Reviews in Biomedical Engineering 2008;1:23-40.

Hinterberger T, Veit R, Strehl U, Trevorrow T, Erb M, Kotchoubey B, Flor H, Birbaumer N. Brain areas activated in fMRI during self-regulation of slow cortical potentials (SCPs). Experimental Brain Research 2003;152:113-122.

Huettel SA, McKeown MJ, Song AW, Hart S, Spencer DD, Allison T, McCarthy G. Linking hemodynamic and electrophysiological measures of brain activity: Evidence from functional MRI and intracranial field potentials. Cerebral Cortex 2004;14:165-173. [PubMed: 14704213]

Jurkiewicz MT, Gaetz WC, Bostan AC, Cheyne D. Post-movement beta rebound is generated in motor cortex: Evidence from neuromagnetic recordings. NeuroImage 2006;32:1281-1289. [PubMed: 16863693]

Kamousi B, Amini AN, He B. Classification of motor imagery by means of cortical current density estimation and von neumann entropy. Journal of Neural Engineering 2007;4:17-25. [PubMed: 17409476]

Kamousi B, Liu Z, He B. Classification of motor imagery tasks for brain-computer interface applications by means of two equivalent dipoles analysis. IEEE Transactions on Neural Systems and Rehabilitation Engineering 2005;13:166-171. [PubMed: 16003895]

Lancaster JL, Woldorff MG, Parsons LM, Liotti M, Freitas CS, Rainey L, Kochunov PV, Nickerson D, Mikiten SA, Fox PT. Automated Talairach Atlas labels for functional brain mapping. Human Brain Mapping 2000;10:120-131. [PubMed: 10912591]

Liu Z, He B. fMRI-EEG integrated cortical source imaging by use of time-variant spatial constraints. Neuroimage 2008;39:1198-1214. [PubMed: 18036833]

Logothetis NK. What we can do and what we cannot do with fMRI. Nature 2008;453:869-878. [PubMed: 18548064]

Logothetis NK, Wandell BA. Interpreting the BOLD signal. Annual Review of Physiology 2004;66:735769.

Logothetis NK, Pauls J, Augath M, Trinath T, Oeltermann A. Neurophysiological investigation of the basis of the fMRI signal. Nature 2001;412:150-157. [PubMed: 11449264]

Lopes da Silva FH, Storm van Leeuwen W. The Cortical source of the alpha rhythm. Neuroscience Letters 1977;6:237-241. [PubMed: 19605058]

Miller KJ, Leuthardt EC, Schalk G, Rao RP, Anderson NR, Moran DW, Miller JW, Ojemann JG. Spectral changes in cortical surface potentials during motor movement. Journal of Neuroscience 2007;27:2424-2432. [PubMed: 17329441]

Moosmann M, Ritter P, Krastel I, Brink A, Thees S, Blankenburg F, Taskin B, Obrig H, Villringer A. Correlates of alpha rhythm in functional magnetic resonance imaging and near infrared spectroscopy. NeuroImage 2003;20:145-158. [PubMed: 14527577]

Mukamel R, Gelbard H, Arieli A, Hasson U, Fried I, Malach R. Coupling between neuronal firing, field potentials, and FMRI in human auditory cortex. Science 2005;309:951-954. [PubMed: 16081741]

Mulert C, Jager L, Schmitt R, Bussfeld P, Pogarell O, Moller HJ, Juckel G, Hegerl U. Integration of fMRI and simultaneous EEG: Towards a comprehensive understanding of localization and time-course of brain activity in target detection. NeuroImage 2004;22:83-94. [PubMed: 15109999]

Muthukumaraswamy SD, Singh KD. Functional decoupling of BOLD and gamma-band amplitudes in human primary visual cortex. Human Brain Mapping 2009;30:2000-2007. [PubMed: 18729078]

Nagai Y, Critchley HD, Featherstone E, Fenwick PB, Trimble MR, Dolan RJ. Brain activity relating to the contingent negative variation: An fMRI investigation. NeuroImage 2004;21:1232-1241. [PubMed: 15050551]

Nunez PL, Wingeier BM, Silberstein RB. Spatial-temporal structures of human alpha rhythms: Theory, microcurrent sources, multiscale measurements, and global binding of local networks. Human Brain Mapping 2001;13:125-164. [PubMed: 11376500]

Oldfield RC. The assessment and analysis of handedness: the Edinburgh inventory. Neuropsychologia 1971;9:97-113. [PubMed: 5146491] 
Pfurtscheller G, Lopes da Silva FH. Event-related EEG/MEG synchronization and desynchronization: Basic principles. Clinical Neurophysiology 1999;110:1842-1857. [PubMed: 10576479]

Qin L, Ding L, He B. Motor imagery classification by means of source analysis for brain-computer interface applications. Journal of Neural Engineering 2004;1:135-141. [PubMed: 15876632]

Olbrich S, Mulert C, Karch S, Trenner M, Leicht G, Pogarell O, Hegerl U. EEG-vigilance and BOLD effect during simultaneous EEG/fMRI measurement. Neuroimage 2009;45:319-332. [PubMed: 19110062]

Ritter P, Moosmann M, Villringer A. Rolandic alpha and beta EEG rhythms' strengths are inversely related to fMRI-BOLD signal in primary somatosensory and motor cortex. Human Brain Mapping 2009;30:1168-1187. [PubMed: 18465747]

Salmelin R, Hamalainen M, Kajola M, Hari R. Functional segregation of movement-related rhythmic activity in the human brain. NeuroImage 1995;2:237-243. [PubMed: 9343608]

Schulz M, Chau W, Graham SJ, McIntosh AR, Ross B, Ishii R, Pantev C. An integrative MEG-fMRI study of the primary somatosensory cortex using cross-modal correspondence analysis. NeuroImage 2004;22:120-133. [PubMed: 15110002]

Singh KD, Barnes GR, Hillebrand A, Forde EM, Williams AL. Task-related changes in cortical synchronization are spatially coincident with the hemodynamic response. NeuroImage 2002;16:103114. [PubMed: 11969322]

Silva LR, Amitai Y, Connors BW. Intrinsic oscillations of neocortex generated by layer 5 pyramidal neurons. Science 1991;251:432-435. [PubMed: 1824881]

Taniguchi M, Kato A, Fujita N, Hirata M, Tanaka H, Kihara T, Ninomiya H, Hirabuki N, Nakamura H, Robinson SE, Cheyne D, Yoshimine T. Movement-related desynchronization of the cerebral cortex studied with spatially filtered magnetoencephalography. NeuroImage 2000;12:298-306. [PubMed: 10944412]

Tallon-Baudry C, Bertrand O, Delpuech C, Permier J. Oscillatory gamma-band (30-70 Hz) activity induced by a visual search task in humans. Journal of Neuroscience 1997;17:722-734. [PubMed: 8987794]

Winterer G, Carver FW, Musso F, Mattay V, Weinberger DR, Coppola R. Complex relationship between BOLD signal and synchronization/desynchronization of human brain MEG oscillations. Human Brain Mapping 2007;28:805-816. [PubMed: 17133396]

Wolpaw JR, McFarland DJ. Control of a two-dimensional movement signal by a noninvasive braincomputer interface in humans. Proceedings of the National Academy of Sciences of the United States of America 2004;101:17849-17854. [PubMed: 15585584]

Yousry TA, Schmid UD, Alkadhi H, Schmidt D, Peraud A, Buettner A, Winkler P. Localization of the motor hand area to a knob on the precentral gyrus. A new landmark. Brain 1997;120 (Pt 1):141-157. [PubMed: 9055804]

Yuan H, Doud A, Gururajan A, He B. Cortical imaging of event-related (de)synchronization during online control of brain-computer interface using minimum-norm estimates in frequency domain. IEEE Transactions on Neural Systems and Rehabilitation Engineering 2008;16:425-431. [PubMed: 18990646] 


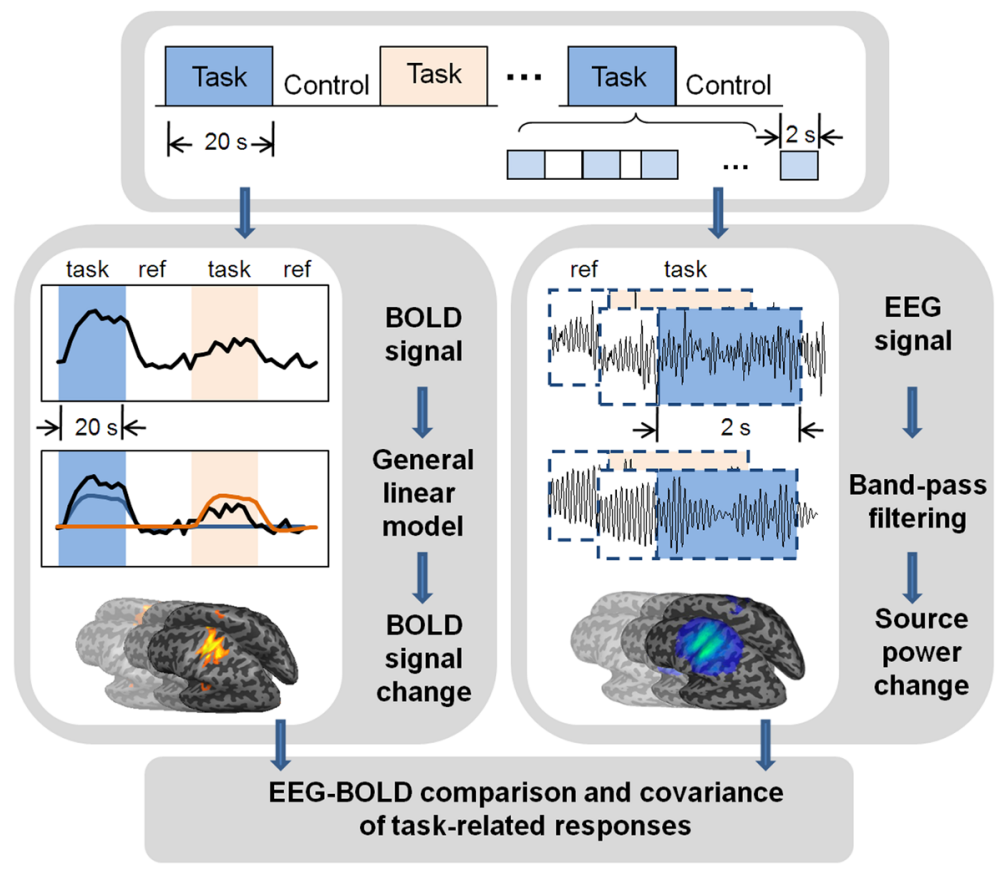

Figure 1.

Schematic illustration of experimental paradigm and data analysis. An example of single-trial EEG activity from the imagination of right hand and its filtered traces $(8 \mathrm{~Hz}-12 \mathrm{~Hz})$ were shown. 
(A) Channel $\mathrm{C} 3$
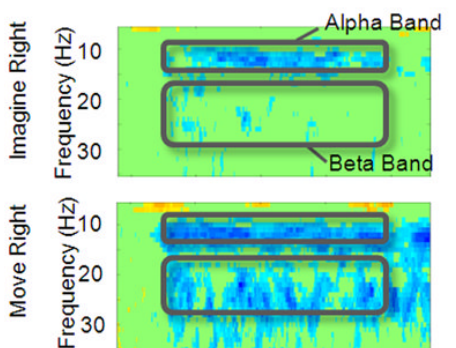

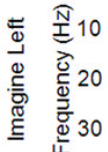
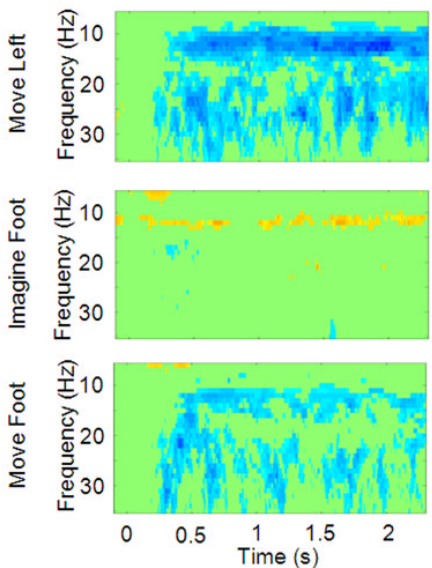

(B) Channel C4
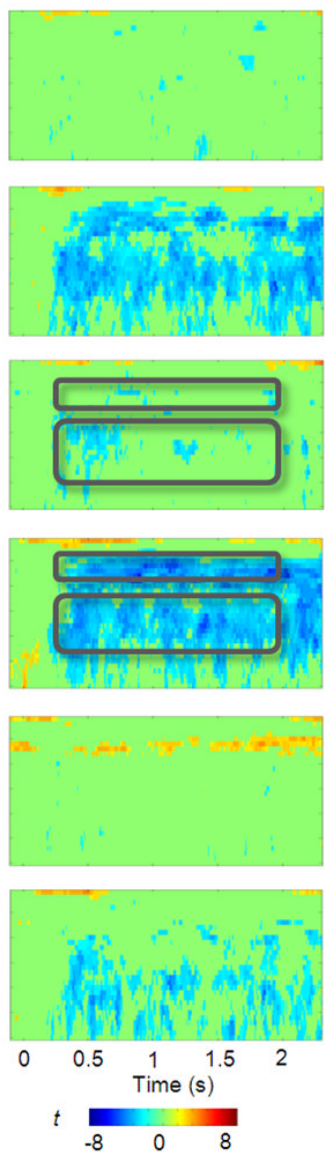

(C) Channel $\mathrm{Cz}$
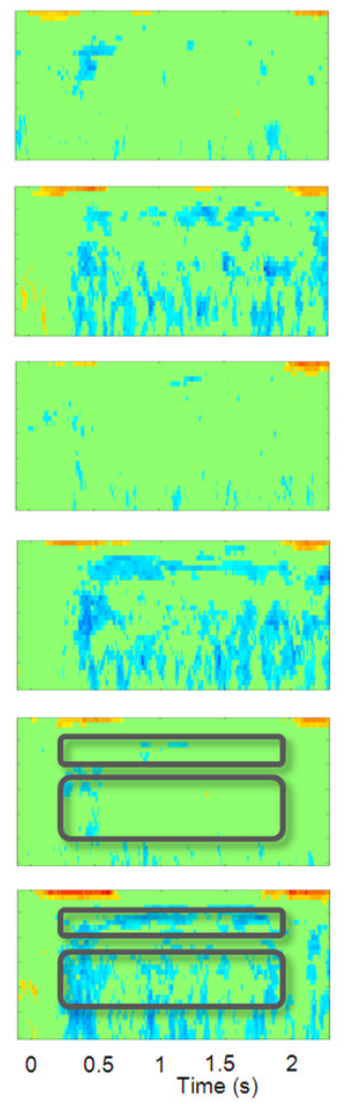

(D) \% Topology
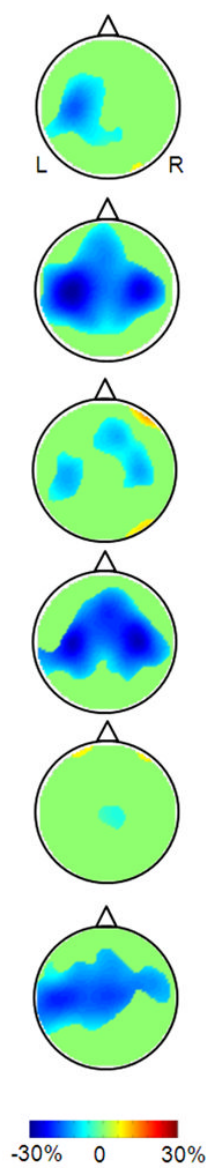

Figure 2.

(A-C) Group Time-Frequency representation of EEG changes from C3, C4 and Cz electrodes. Power changes relative to baseline are depicted as $t$ statistic thresholded by $p<0.05(|t|>2.18$, uncorrected for multiple comparison). Black squares in each plot indicate the alpha- and betafrequency bands of interest. (D) Scalp topology of relative power changes in the beta band $(p<0.05$, uncorrected). 


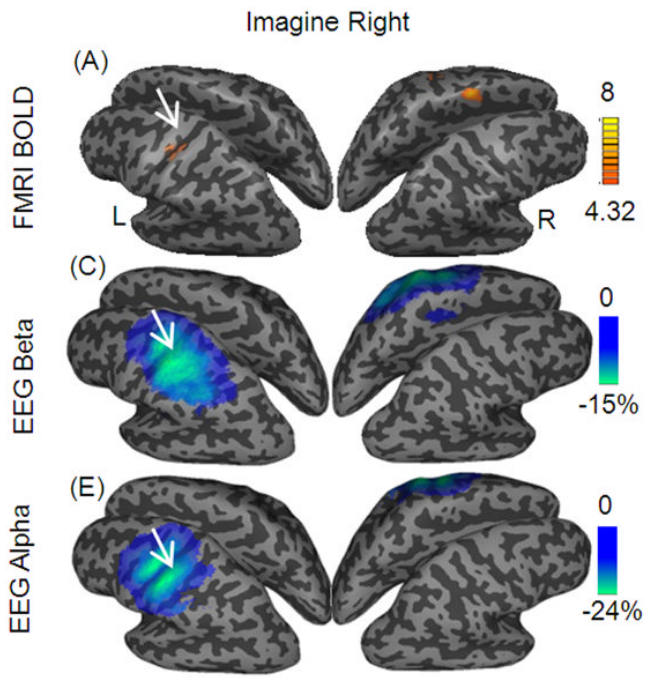

(G) Imagine and Move Right Hand, Beta vs BOLD

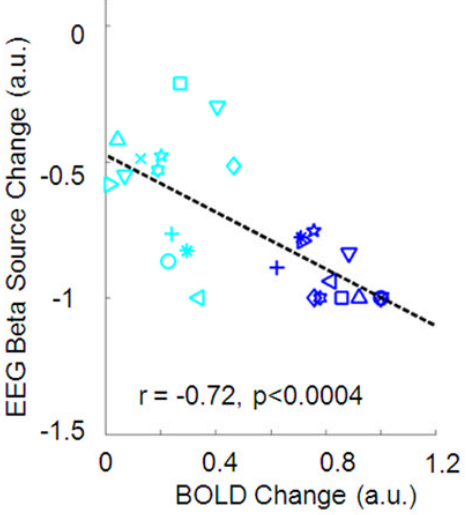

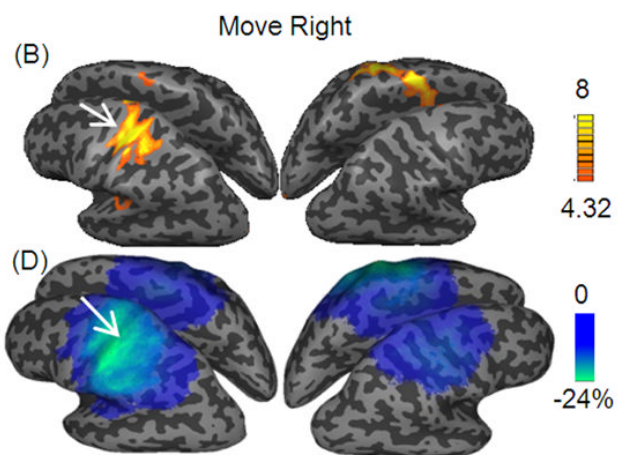

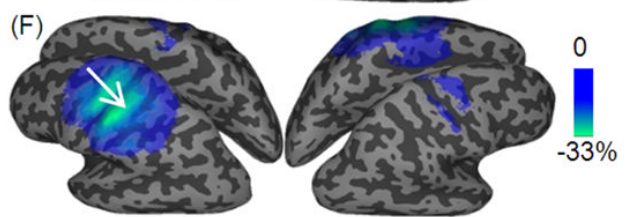

(H) Imagine and Move Right Hand, Alpha vs BOLD

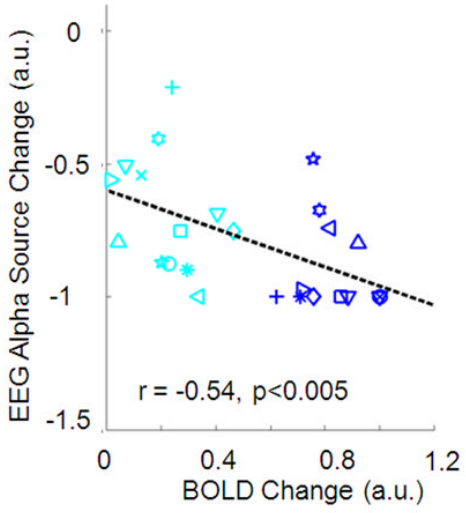

Figure 3.

EEG and fMRI results during imagination and movement of right hand. (A-F) shows the group maps on an inflated cortical surface with white arrows indicating the peak changes at the primary sensorimotor cortex. (A-B) shows BOLD $t$ statistic maps $(p<0.001$, uncorrected for multiple comparison). ( $\mathrm{C}-\mathrm{F})$ shows average percent change $(p<0.001$, uncorrected) in beta and alpha bands. $(\mathrm{G})$ shows the covariation between quantified responses in EEG beta and BOLD at the contralateral hand ROI from the pooled imagination (cyan) and movement (blue) tasks. (H) shows a similar linear relation between EEG alpha and BOLD responses. Different symbols in $(\mathrm{G}-\mathrm{H})$ indicate responses from different individual subjects. 

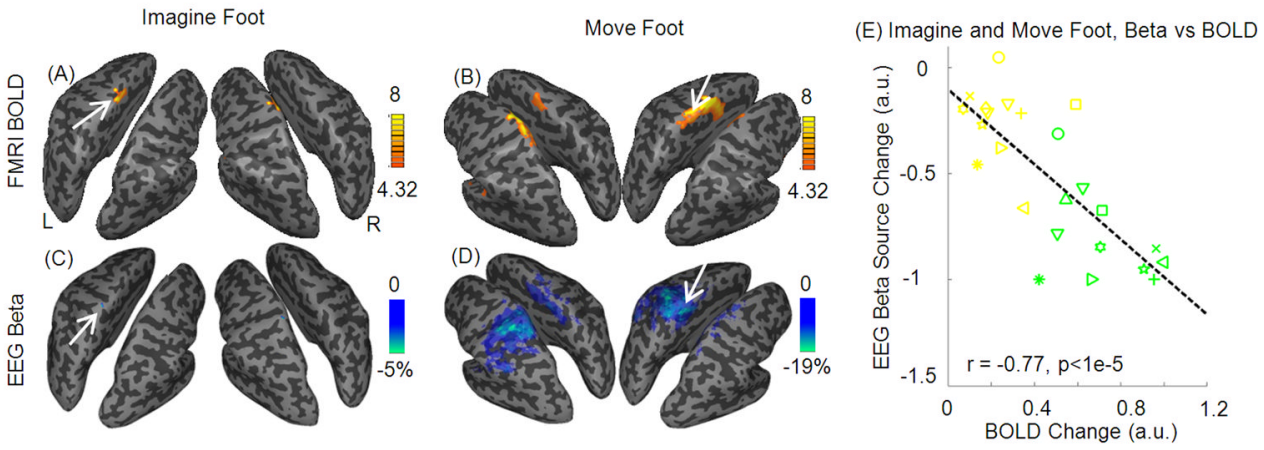

Figure 4.

EEG and fMRI results during imagination and movement of right foot. (A-D) shows the group maps on an inflated cortical surface with white arrows indicating the peak changes at the primary sensorimotor cortex. (A-B) shows BOLD $t$ statistic maps $(p<0.001$, uncorrected for multiple comparison). (C-D) shows average percent change ( $p<0.001$, uncorrected) in beta band. (E) shows the co-varied changes of EEG beta and BOLD at the foot ROI from pooled quantities of imagination (yellow) and movement (green). Different symbols in (E) indicate responses from different individual subjects. 

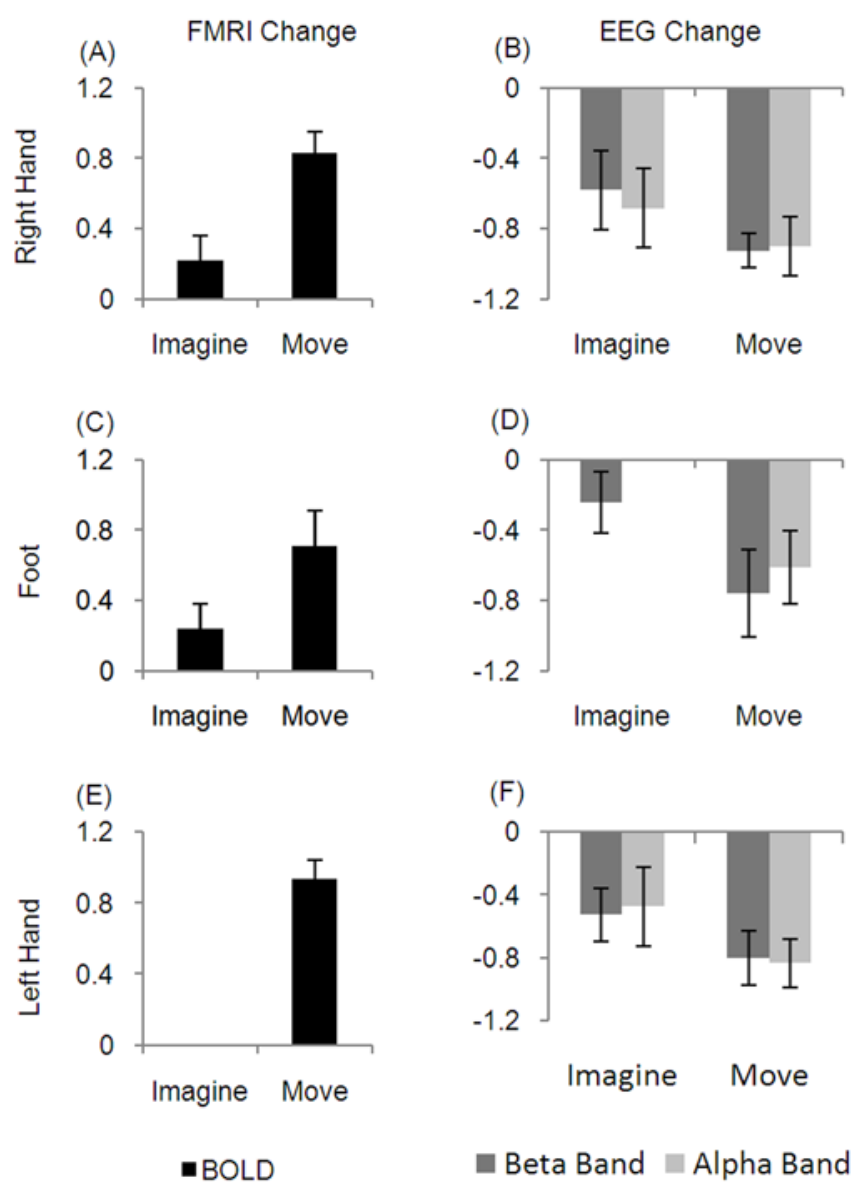

Figure 5.

Average changes of fMRI (A, C and E) and EEG (B, D, and F) in regions of interest for all task conditions under threshold $p<0.001$ (uncorrected for multiple comparison). Notice that no regions were found in BOLD maps for imagination of left hand, or EEG alpha maps for imagination of right foot. 

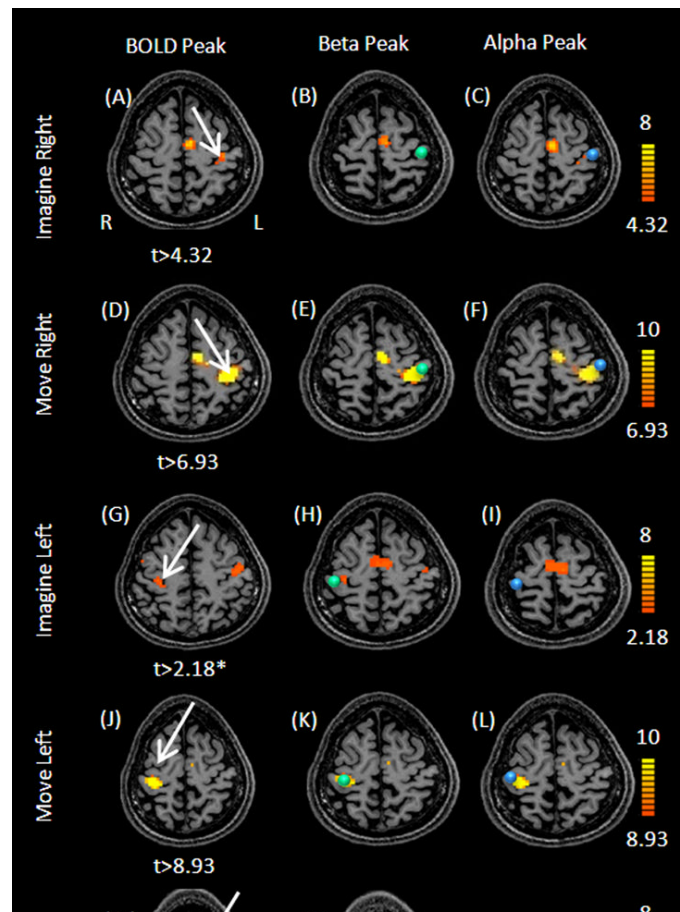

(K)
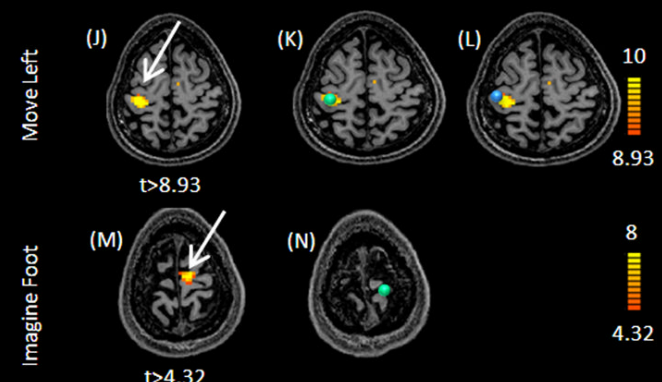

(M)

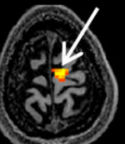

(N)
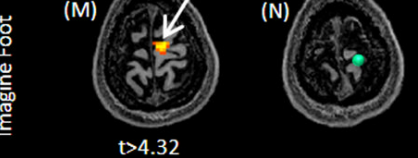

艹 (0)

$$
\mathrm{t}>4.32
$$

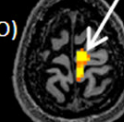

(P)

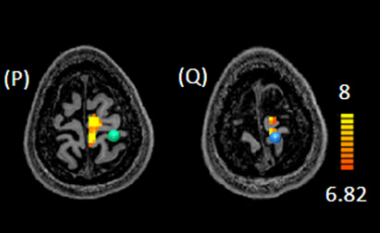

Figure 6.

Locations of maximum changes in EEG alpha (blue balls), beta (green balls) and BOLD (white arrows), superimposed on axial MRI images with BOLD $t$ statistic maps. The thresholds for BOLD maps were adjusted for each task condition to show where the peaks were. $t$-value $=$ 2.18 ( $p=0.05$, uncorrected), 4.32 ( $p=0.001$, uncorrected). 


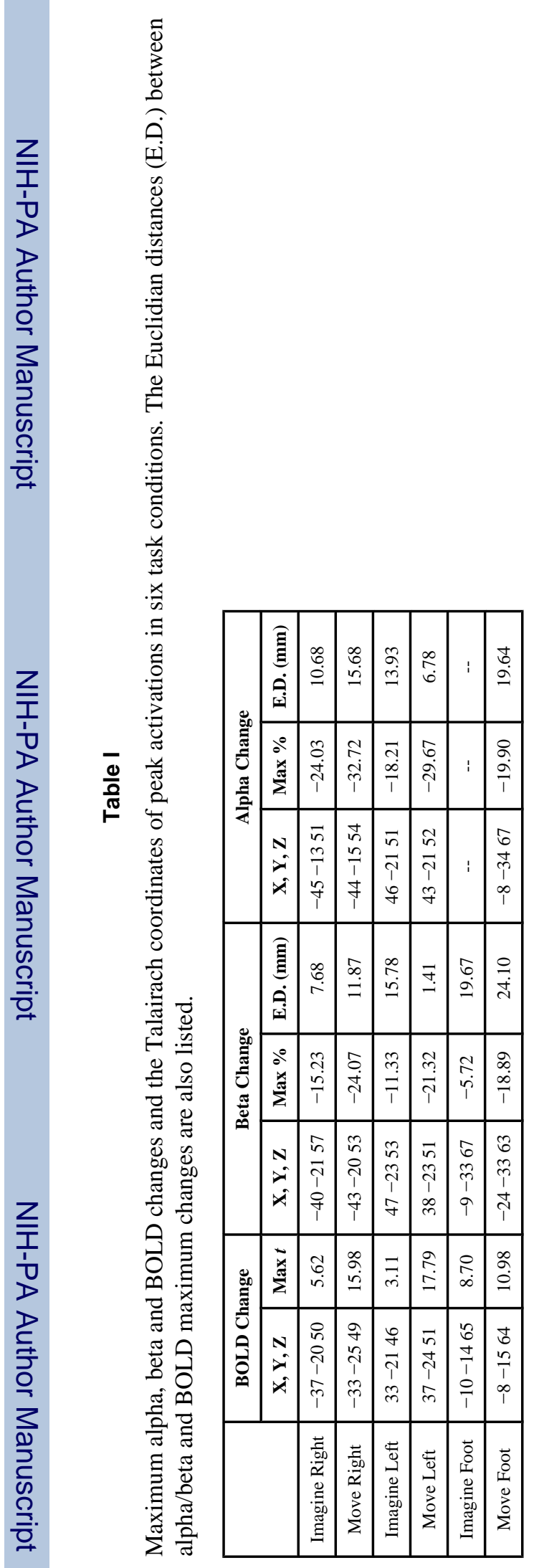

Neuroimage. Author manuscript; available in PMC 2011 February 1. 